\title{
Market Access: More for Less from Clinical Trials to Multi-criteria Decision Analysis
}

Josep M Garcia-Alamino*

Nuffield Department of Primary Care Health Sciences, University of Oxford, Oxford, UK

*Corresponding author: Josep M Garcia-Alamino, Nuffield Department of Primary Care Health Sciences, University of Oxford, Oxford, UK, E-mail: jose.garciaalamino@kellogg.ox.ac.uk

Received date: May 26, 2017; Accepted date: June 01, 2017; Published date: June 05, 2017

Copyright: (c) 2017 Garcia-Alamino JM. This is an open-access article distributed under the terms of the Creative Commons Attribution License, which permits unrestricted use, distribution, and reproduction in any medium, provided the original author and source are credited.

\section{Editorial}

Today, market access should be guided by the presence of innovative products that can be incorporated into health-care systems or national health services, thereby guaranteeing their sustainability. However, the current reality is that the introduction of a new drug or medical device often ends up being a strategy for replacing an old product with a more innovative one. This change occurs without increasing the purchase price of the new product. It is what someone has called "more for less".

Market access, reimbursement and price should be more dependent on the added value that new treatments or medical devices bring to the patient and society.

Therefore, the different stakeholders should collaborate to find the balance that benefits the patient in the earliest way, so that the health system can assume it at a sustainable cost and so that the medical industry obtains a benefit that allows it to recover the investment during the lifetime of the patent. Articulating these elements is not easy, because often one of the parties may not feel satisfied.

Fortunately, market access seems to shift from a "pay by volume" model to a "payment by clinical results" model. These most innovative purchase models have a major advantage for the gatekeeper (Minister of Health, private hospitals) because only pay what works in their population and the economic benefit is also obtained immediately, in addition to not having to assume a high risk at the initial time of purchase. This new model of purchase is even more interesting for the gatekeeper than the classic cost-effectiveness models in which the benefit is obtained in the long term or even, sometimes, not even seen. At the moment any drug or medical device if you want to get access to the market is important to present an innovation and really this innovation brings value. This value should translate into clinical efficacy, route of administration, improvement of compliance by the patient and improvement in quality of life, among others. However, evaluating health outcomes is not an easy task, and even less so when evaluating under real conditions of current clinical practice.

The classic evaluation of evidence through randomised controlled trials continues to be a tool that provides us with valid results on the effectiveness of a product in ideal situations (i.e. controlled) and is considered a tool with a high quality of evidence, very close to cumulative meta-analysis. However, it is widely accepted that results obtained in a randomised controlled trial or a meta-analysis may not correlate later with the results obtained in practice. This is due to several reasons: 1) Differences between the sample analysed (i.e., restrictive inclusion criteria) and the actual population; 2) Issues in the application of the drug or medical device (e.g. experience of the physician, surgeon or nurse); 3) Lack of compliance by the patient or relatives; 4) Other reasons. This makes it difficult for clinical trial results to be the only objective test to assess the effectiveness of a new product and its market access. The gatekeeper (regulatory agencies, national health service) no longer relies solely on the results of the clinical trial, but needs to know how that product will behave in the population for which health care should be provided. In the case of medical devices, the classic performance of a trial with randomisation and blinded evaluation is not always possible due to the characteristics of the intervention. For this reason, and among other reasons, several stakeholders have proposed the use of data closer to the real environment to evaluate effectiveness and monitor the results. One way to do this has been to employ "Real-World Evidence" (RWE), which considers information from observational studies, electronic health records, disease registries and even data gathered through personal devices. Although this information may have the biases of non-comparative or randomised studies, it provides very valuable information to enable approximation of the performance of a product in real clinical practice. In fact, for some regulatory agencies the key to market access is to demonstrate value in real practice. There are experiences in which agencies allow access and payment only when the product demonstrates results in their populations (payment by results) [1]. However, monitoring the clinical results obtained to perform this payment by results is difficult. One of the classic shortcomings of health systems is the lack of useful sources of clinical information. Most of the electronic data stored and available over time are administrative (i.e. billing) and the clinical data generated daily are not available for analysis over time. Therefore, big data could contribute in a very important way to improving this situation by providing a great volume of data, as long as the data provided by the big data are debugged and provide relevant information, and are not limited to providing a large volume of data. Large case series (i.e., observational studies) pooling their results, for example, using proportional metaanalysis can provide valuable information in this field.

However, the aforementioned tools mainly contribute to providing information in the clinical dimension, but not at the level of burden of disease, costs, values and the preferences of the various stakeholders (patients, providers, payers). Therefore, in addition to all clinical evidence, it is also important to incorporate the results of pharmacoeconomics and impact on quality of life. Although all this information is available, it is difficult to integrate and analyse it in a global scenario. In order to improve this scenario, facilitating clinical decision-making and access to the market of those therapies or devices of greater benefit, some agencies or other institutions should begin to work with the Multi-criteria Decision Analysis (MCDA) tool.

MCDA is a valuable tool for facing many complex decisions involving multiple-criteria goals or objectives of a conflicting nature [2]. 
Citation: Garcia-Alamino JM (2017) Market Access: More for Less from Clinical Trials to Multi-criteria Decision Analysis. Health Econ Outcome Res 3: e107. DOI: 10.4172/2471-268X.1000e107

Page 2 of 2

MCDA allows decision-makers to structure clinical evidence, quality of life data, and health economics data available for a drug or a medical device, and provides a visual guide for decision-making. The implementation of the MCDA tool requires a series of structured and pre-established steps. Firstly, the criteria to be evaluated (clinical end points, mode of administration, among others) are listed along with all the available alternatives (treatments or medical devices). The next step is to assign a weight to each criterion, for example 0 to 100 , to indicate the relative importance of each one. Performance scores are then analysed and, in this case, experts evaluate how the various alternatives are adapted to each criterion. The aggregation of the criteria and the performance score will give us the value of the best treatment alternative. Patients, providers and payers should all be involved throughout the process.
To maintain the development, innovation and incorporation of products with added value to populations it is important to look for formulas to move from a model of payment by volume to a model of payment by results that benefits all the parties involved.

\section{References}

1. Clopes A, Gasol M, Cajal R, Segú L, Crespo R, et al. (2017) Financial consequences of a payment-by-results scheme in Catalonia: gefitinib in advanced EGFR-mutation positive non-small-cell lung cancer. J Med Econ 20: 1-7.

2. Migliore A, Integlia D, Bizzi E, Piaggio T (2015) Is it the time to rethink clinical decision-making strategies? From a single clinical outcome evaluation to a Clinical Multi-criteria Decision Assessment (CMDA). Med Hypothes 85: 433-440. 